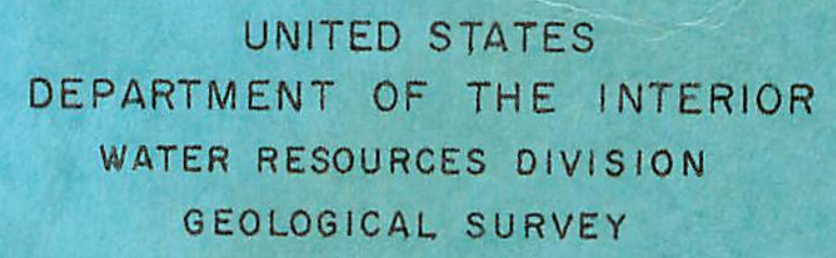

\title{
SUMMER BASE-FLOW \\ RECESSION CURVES FOR IOWA STREAMS
}

\author{
By \\ Carroll W. Saboe \\ Hydraulic Engineer, USGS
}

Prepared in Cooperation with

THE IOWA NATURAL. RESOURCES COUNCIL

\author{
Open-file Report \\ $66-a$ \\ lowa City, lowa \\ July 1966
}


UNITED STATES

DEPARTMENT OF THE INTERIOR

WATER RESOURCES DIVISION

GEOLOGICAL SURVEY

\title{
SUMMER BASE-FLOW \\ RECESSION CURVES FOR IOWA STREAMS
}

\author{
By \\ Carroll W. Saboe \\ Hydroulic Engineer, USGS \\ Prepared in Cooperation with
}

THE IOWA NATURAL RESOURCES COUNCIL

Open-file Report

lowa City, lowa

July 1966 
IOHA NATURAL RESOURCES COUNCIL

H. Garland Hershey, Iowa City, Chairman Stanley I. Haynes, Mason City, Vice-Chatrman Louis P. Culver, Dunlap, Secretary

J. Robert Downing, Indianola

J. W. Howe, Iowa City

William G. Murray, Ames

Clifford M. Naser, Fort Dodge

J. Justin Rogers, Spirit Lake

L. Guy Young, Bedford

Othie R. McMurry; Director

R. G. Bullard, Water Commissioner

UNITED STATES DEPARTMENT OF INTERIOR

GEOLOGICAL SURVEY

Williem T. Pecora, Director

WATER RESOURCES DIVISION

E. L. Hendricks, Chief Hydrologist

M. R. Williams, Chief, Surface Water Branch

S. W. Wítala, Iowa District Engineer 


\section{CONTENTS}

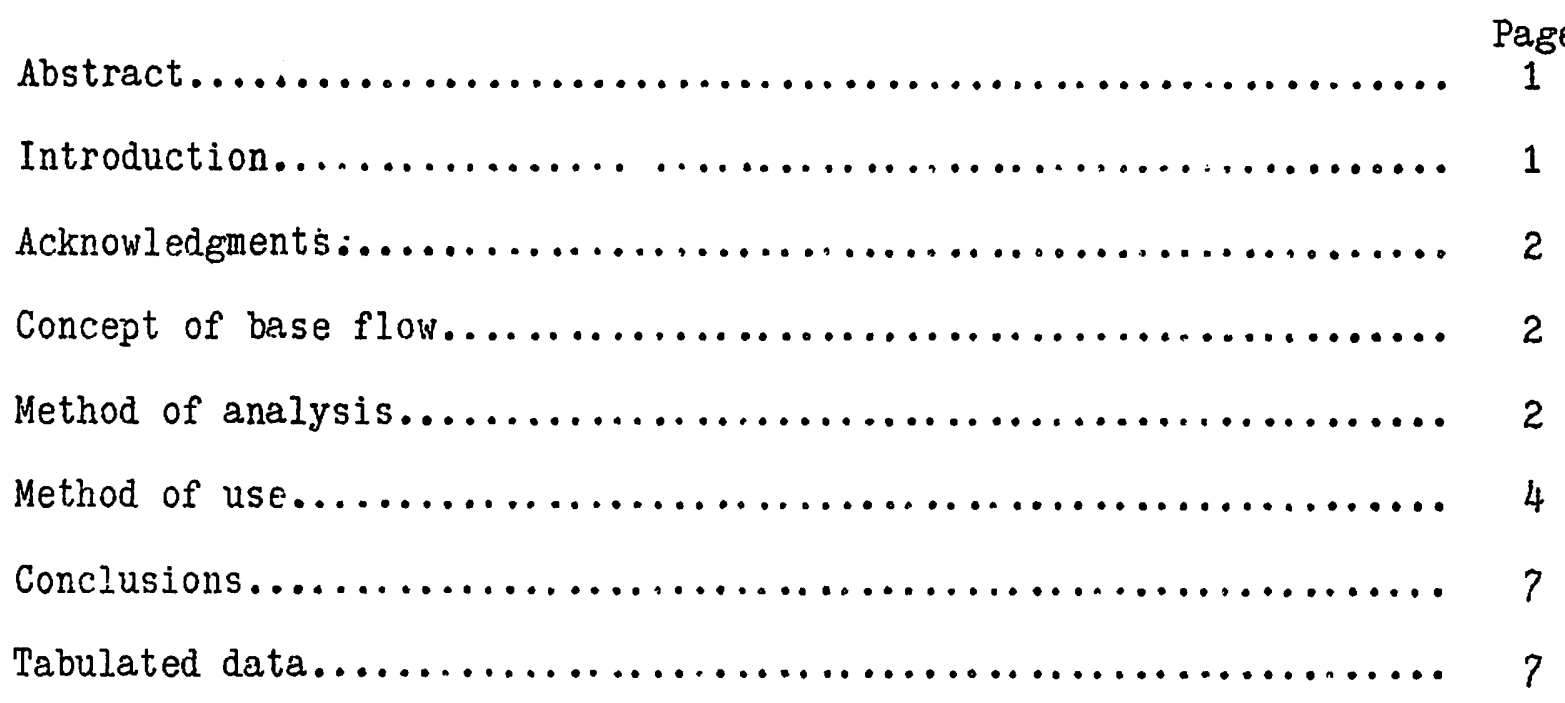

\section{ILIUSTRATIONS}

Figure 1. Hap of Iowa showing location of lake and stream-gaging stations operated by the U. S. Geological Survey in cooperation with other agencies.................... 3

Figure 2. Relation between selected starting base flow and that ten days later, Cedar River at Waterloo, Iowa.............

Figure 3. Base-flow recession curves, Cedar River at Waterloo,

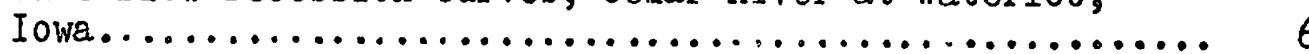

TABLTS

Table 1. Plotting data for base-flow recession curves. 


\section{ABSTRACT}

Base-flow recession curves for the summer months (June through September) were developed in this study for gaging stations on interior Iowa streams having five or more years of record. The tabulated data enables the user, starting with a known base flow at a gage, to estimate base flows for up to 20 days in the future. Rainfall during the period of the forecast will require that a now estimate be made after the stream again reaches base flow.

\section{INTRODUCTION}

Forecasts of probable future surface-water flows are required for many purposes. Among these are (1) administering water laws, particularly those concerned with withdrawal use for irrigation, (2) evaluating streamflow available for municipal and domestic water supply to determine possible need for supplemental supplies, and (3) evaluating adequacy of streamflow for waste dilution to determine if wastes should be temporarily stored or accelerated treatment initiated.

This report has been prepared to provide a means for making shortterm forecasts of base flow of Iowa streams at gaging stations. Baseflow recession curves are used for this purpose. These curves define the relation between base-flow discharge and time. Reliable estimates of future flows up to 20 days in advance are possible when no significant rainfall occurs in the interim period.

The data presented in this report are based on records of streamflow that have been collected at gaging stations on interior Iowa. streams. 
The period of record available at each gaging station was used in preparing the base-flow recession data. In general, streamflow records shorter than 5 years were inadequate for this analysis.

\section{ACKITOVLEDGHENTS}

This study was undertaken at the request of the Iowa Natural Resources Council as a part of the cooperative program with the U. S. Geological Survey. The streamflow records utilized were collected by the U. S. Geological Survey in cooperation with Federal, State, local, and private agencies. These agencies are listed in the annual series of streamflow reports issued by the U. S. Geological Survey.

The work of analyzing the records and preparing the report was done at the District Office, U. S. Geological Survey, Iowa City, Iowa.

\section{CONCEPT OF BASE FLOW}

Base flow includes the ground-water component of total flow plus a component of flow known as interflow, which is water that moves in the zone above the water table and reaches the stream channels some time after surface runoff has ceasad. Base flow maintains streamflos during rainless jeriods. For very small basins, base floil could occur a day or two after a rain.

\section{VETHOD OF AHAIYSIS}

For this report, hyarographs of total flow were analyzed for the months of June through Septerber for each year of record at all gaging stations. A map showing the location of the gaging stations in Iowa appears as figure 1. During periods when there is no surface runoff, the 


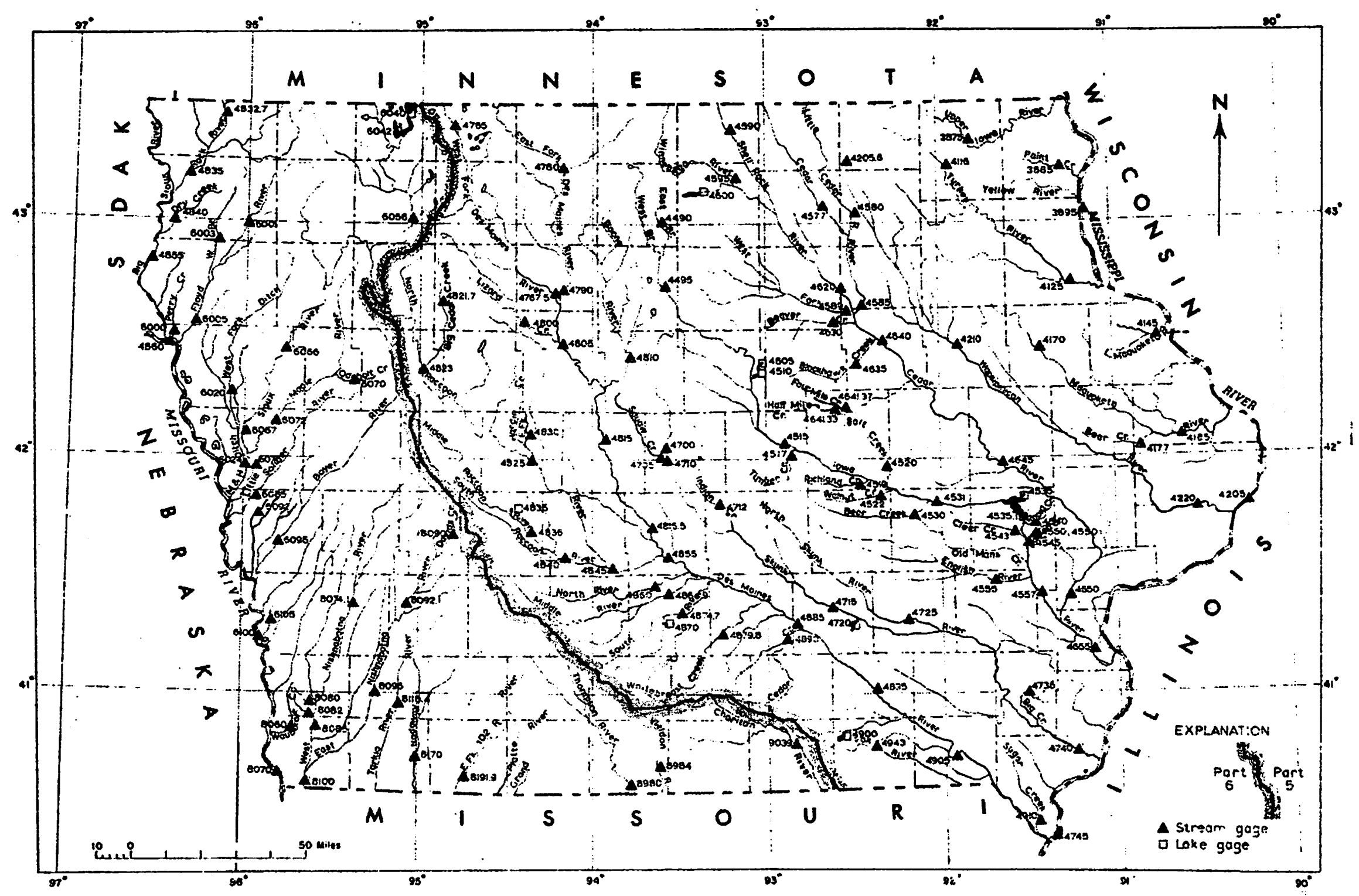

Figure 1.--Map of Iowa showing location of lake and stream gaging stations operated by the U. S. Geological Survey in cooperation with other agencies. 
rate of recession appears to be essentially constant for any given baseflow discharge during the June-through-September period. This phenomenon is the basis for development of the base-flow recession eurve.

The following procedures were used to derive the base-flow recession values:

1. For base-flow periods, discharge on a beginning day and the discharge 10 days later were tabulated. A 5-day interval was used on small streams.

2. Values of beginning-day discharge were plotted as abscissa against discharge 10 days later as ordinate on logarithmic paper. A line was drawn averaging the points (line $A, f i s .2)$. A second line was drawn ignoring all points except those farthest to the right (1ine B, fig. 2). Iine A represents the average recession rate and line $B$ represents approximately the maximum recession rate defined by the data.

3. Curves $A$ and $B$ (fig. 2) were then used to develop the curves of figure 3.

The base-flow recession data listed in table 1 were obtained from curves prepared as explained above. Curves similar to those of figure 3 can be plotted using the tabulated station data. All values of discharge are in cubic feet per second (cfs).

\section{METHOD OF USE}

For each gagine station, valued for two base-flow recession curves are shown. Curve "A" represents the average experience that has occurred at the gaging station. Use of curve "A: is recommended when evapotranspiration losses are norul or average. Curve "B" represents a more severe condition that may occur when evapotranspiration losses are abnormally large. 


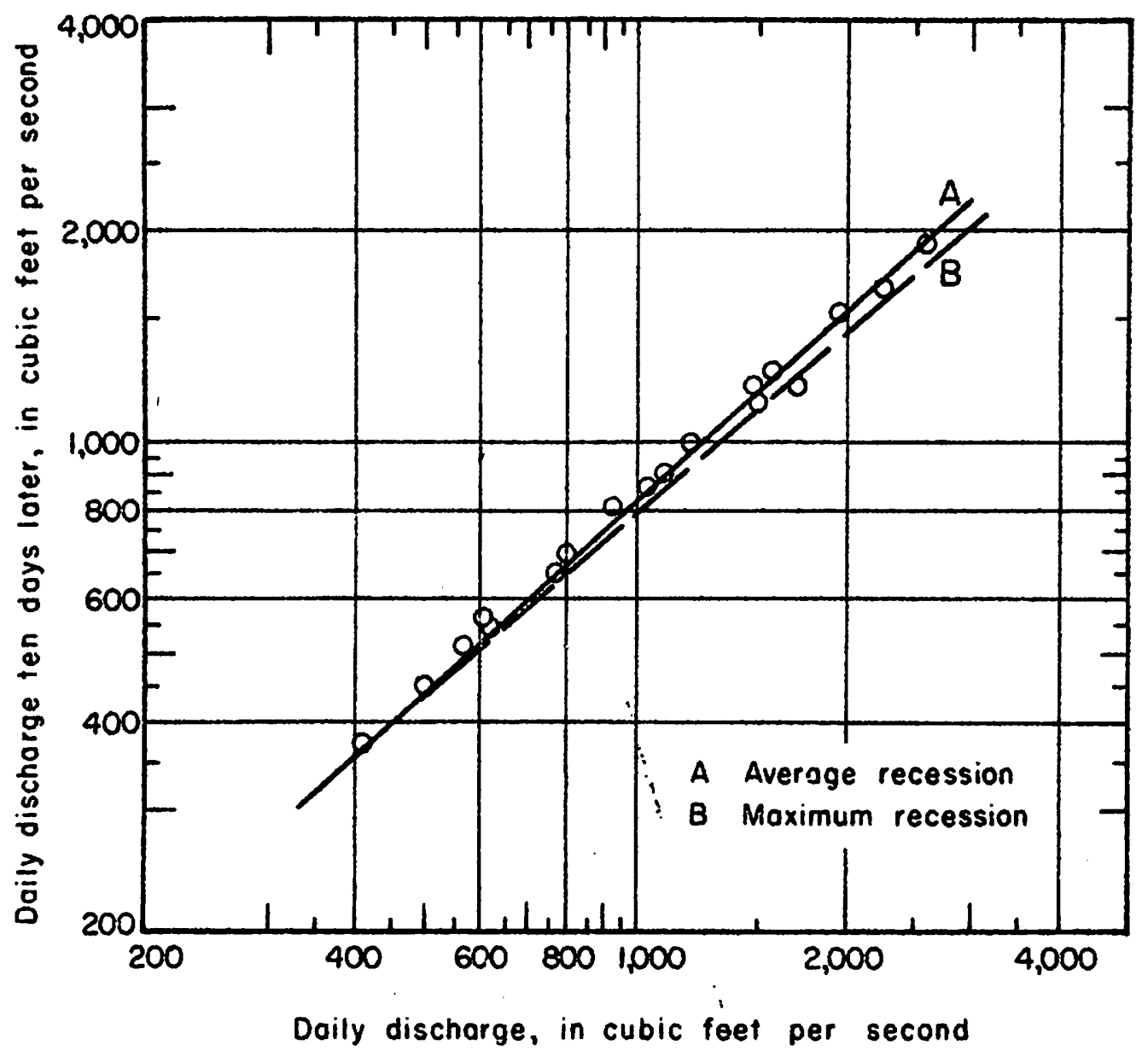

Figure 2. Relation between base flow on a selected day and that ten days later, Cedar River at Waterloo, lowa $(5,146 \mathrm{sq} \mathrm{mi})$. 


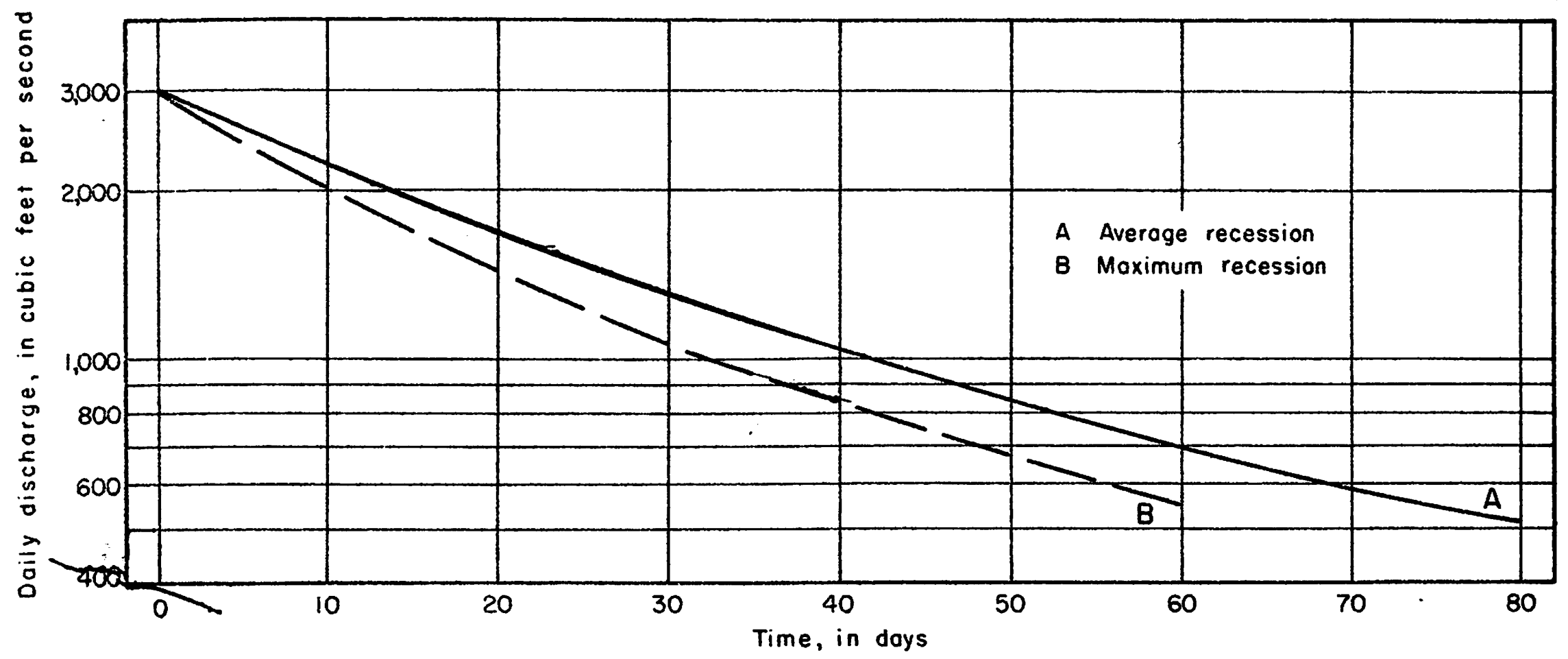

Figure 3. Base-flow recession curves, Cedar River of Waterloo, lowa. 
Other factors, undefined by this study, may also affect the recession rate. The steeper recession rate defined by curve " $B$ " probably occurs during prolonged periods of excessively hot and windy weather. The curves are valid for periods of no rainfall and for the summer season only.

As an example, when the base-flow discharge at the Waterloo gaging station is $1,300 \mathrm{cfs}$, the estimated discharge 20 days later would be 835 cfs using curve "A", figure 3. If conditions indicate the use of curve "B", the corresponding discharge discharge would be 780 cfs 20 days later.

\section{CONCLUSIONS}

Base-flow recession curves are useful tools to estimate future discharges in a stream. However, since only short segments of base flow were used in their derivation, the length of the period of estimate should, in general, be less than 20 days. As additional low-flow records become available, the base-flow recession curves will become better defined.

\section{TABULATED DATA}

The following table (table 1) contains the data for plotting curves similar to figure 3. The gaging stations are identified by number and name. Additional information on the stations may be obtained from the latest report, "Water Resources Data for Iowa, Part 1, Surface Water Records" published yearly by the U. S. Geological Survey, The order of listing of the gaging stations in table $I$ is the same as in the above publication. 
Table 1. Plotting data for base-flow recession curves .

\begin{tabular}{|c|c|c|c|c|c|c|c|c|c|c|}
\hline \multirow{3}{*}{$\begin{array}{l}\text { Time } \\
\text { in } \\
\text { days }\end{array}$} & \multirow{2}{*}{\multicolumn{2}{|c|}{$\begin{array}{l}\text { Upper Iowa River } \\
\text { at Decorah } \\
511 \mathrm{sq} \mathrm{mi}\end{array}$}} & \multicolumn{2}{|c|}{$\begin{array}{c}5-3885 \\
\text { Paint Creek at } \\
\text { Waterville }\end{array}$} & \multicolumn{2}{|c|}{$\begin{array}{c}5-4116 \\
\text { Turkey River at } \\
\text { Spillville }\end{array}$} & \multicolumn{2}{|c|}{$\begin{array}{c}5-4125 \\
\text { Turkey River } \\
\text { at Garber }\end{array}$} & \multicolumn{2}{|c|}{$\begin{array}{c}5-4145 \\
\text { Little Naquoketa River } \\
\text { near Durango }\end{array}$} \\
\hline & & & 42.8 & $\mathrm{mi}$ & 177 & $\mathrm{mi}$ & 1545 & $\mathrm{mi}$ & $130 \mathrm{~s}$ & mi \\
\hline & $A$ & B & A & $B$ & A & $B$ & A & B & A & $B$ \\
\hline $\begin{array}{r}0 \\
5 \\
10 \\
15 \\
20 \\
25 \\
30 \\
35 \\
40 \\
45 \\
50 \\
55 \\
60 \\
65 \\
70 \\
75 \\
80 \\
85 \\
90 \\
95 \\
100\end{array}$ & $\begin{array}{l}500 \\
420 \\
354 \\
296 \\
250 \\
210 \\
177 \\
150 \\
126 \\
107 \\
91 \\
77 \\
66\end{array}$ & $\begin{array}{r}500 \\
385 \\
296 \\
228 \\
174 \\
133 \\
103 \\
78 \\
60\end{array}$ & $\begin{array}{r}20.0 \\
17.0 \\
14.4 \\
12.2 \\
10.4 \\
8.9 \\
7.7 \\
6.6 \\
5.7 \\
4.9 \\
4.2 \\
3.7 \\
3.2 \\
2.8 \\
2.4 \\
2.1 \\
1.8 \\
1.6 \\
1.4\end{array}$ & $\begin{array}{r}20.0 \\
14.1 \\
9.9 \\
7.1 \\
5.2 \\
3.8 \\
2.8 \\
2.0 \\
1.5\end{array}$ & $\begin{array}{r}100 \\
85.0 \\
72.0 \\
62.0 \\
54.5 \\
47.5 \\
42.0 \\
36 . ? \\
32.5 \\
29.0 \\
26.0 \\
23.4 \\
21.2 \\
19.2 \\
17.6 \\
16.0 \\
14.7 \\
13.7 \\
12.8\end{array}$ & $\begin{array}{l}100 \\
67.0 \\
48.0 \\
36.0 \\
28.4 \\
23.0 \\
19.0 \\
16.2 \\
14.2 \\
12.7 \\
11.5\end{array}$ & $\begin{array}{r}800 \\
690 \\
585 \\
500 \\
435 \\
380 \\
335 \\
294 \\
258 \\
228 \\
204 \\
182 \\
164 \\
147 \\
133 \\
120 \\
109 \\
99 \\
90 \\
82 \\
75\end{array}$ & $\begin{array}{r}800 \\
640 \\
505 \\
410 \\
340 \\
284 \\
237 \\
200 \\
172 \\
150 \\
130 \\
114 \\
101 \\
90 \\
80\end{array}$ & $\begin{array}{r}60.0 \\
51.0 \\
43.0 \\
36.4 \\
30.5 \\
25.6 \\
21.6 \\
18.2 \\
15.2 \\
12.8 \\
10.8 \\
9.0 \\
7.6\end{array}$ & $\begin{array}{r}60.0 \\
44.5 \\
33.6 \\
25.4 \\
19.2 \\
14.4 \\
10.8 \\
8.2\end{array}$ \\
\hline
\end{tabular}

* Use curve $A$ when evapotranspiration losses are average or normal. Use curve $B$ during excessively jot, windy weather. 
Table 1. Plotting data for base-flow recession curves -- continued

\begin{tabular}{|c|c|c|c|c|c|c|c|c|c|c|}
\hline \multirow{4}{*}{$\begin{array}{l}\text { Time } \\
\text { in } \\
\text { days }\end{array}$} & \multicolumn{10}{|c|}{ Gaging station number and name, drainage area, and discharge in cfs for curves $\lambda$ and $B *$} \\
\hline & \multirow{2}{*}{\multicolumn{2}{|c|}{$\begin{array}{l}\begin{array}{c}5-4170 \\
\text { laquoketa River } \\
\text { near Hanchester }\end{array} \\
305 \mathrm{sq} \mathrm{mi}\end{array}$}} & \multirow{2}{*}{\multicolumn{2}{|c|}{$\frac{\begin{array}{c}5-4177 \\
\text { Bear Creek near } \\
\text { Honmouth }\end{array}}{61.3 \mathrm{sq} \mathrm{mi}}$}} & \multirow{2}{*}{\multicolumn{2}{|c|}{$\begin{array}{l}\begin{array}{l}5-4185 \\
\text { Haquoketa River } \\
\text { near liaquoketa }\end{array} \\
15.53 \text { sq ini }\end{array}$}} & \multirow{2}{*}{\multicolumn{2}{|c|}{$\begin{array}{c}\begin{array}{c}5-4205.6 \\
\text { Wapsipinicon River } \\
\text { near Elma }\end{array} \\
95.2 \mathrm{sq} \mathrm{mi}\end{array}$}} & \multicolumn{2}{|c|}{$\begin{array}{l}5-4210 \\
\text { Hapsipinicon River } \\
\text { at Independence }\end{array}$} \\
\hline & & & & & & & & & $1048 \mathrm{~s}$ & $\mathrm{mi}$ \\
\hline & A & B & A & $B$ & A & B & $\bar{A}$ & $\bar{B}$ & $\bar{A}$ & $\bar{B}$ \\
\hline $\begin{array}{r}0 \\
5 \\
10 \\
15 \\
20 \\
25 \\
30 \\
35 \\
40 \\
45 \\
50 \\
55 \\
60 \\
65 \\
70 \\
75 \\
80 \\
85 \\
90 \\
95 \\
100\end{array}$ & $\begin{array}{r}400 \\
290 \\
216 \\
165 \\
130 \\
104 \\
86 \\
72 \\
61 \\
53 \\
46 \\
41 \\
37\end{array}$ & $\begin{array}{r}400 \\
240 \\
157 \\
109 \\
79 \\
61 \\
48 \\
40 \\
34\end{array}$ & $\begin{array}{r}30.0 \\
24.0 \\
18.9 \\
15.0 \\
11.9 \\
9.4 \\
7.5 \\
5.9 \\
4.7 \\
3.7 \\
3.0 \\
2.4 \\
1.9\end{array}$ & $\begin{array}{r}30.0 \\
22.3 \\
16.6 \\
12.2 \\
8.9 \\
6.5 \\
4.7 \\
3.4 \\
2.4 \\
1.8\end{array}$ & $\begin{array}{r}1500 \\
1210 \\
990 \\
820 \\
700 \\
595 \\
510 \\
440 \\
385 \\
345 \\
310 \\
230 \\
255 \\
235 \\
215 \\
200 \\
185\end{array}$ & $\begin{array}{r}1500 \\
1190 \\
950 \\
760 \\
610 \\
490 \\
400 \\
325 \\
265 \\
220 \\
180\end{array}$ & $\begin{array}{r}20.0 \\
16.3 \\
13.4 \\
11.2 \\
9.5 \\
8.2 \\
7.2 \\
6.3 \\
5.6 \\
5.0 \\
4.6 \\
4.1 \\
3.7\end{array}$ & $\begin{array}{r}20.0 \\
15.0 \\
11.4 \\
9.0 \\
7.2 \\
5.9 \\
4.9 \\
4.1 \\
3.5 \\
2.9\end{array}$ & $\begin{array}{l}230 \\
200 \\
178 \\
157 \\
141 \\
125 \\
111 \\
100 \\
89.5 \\
81.0 \\
73.0 \\
66.0 \\
60.0 \\
54.5 \\
49.5 \\
45.5 \\
42.0 \\
39.0 \\
36.0 \\
33.0 \\
30.0\end{array}$ & $\begin{array}{l}230 \\
197 \\
166 \\
140 \\
118 \\
102 \\
87.0 \\
74.5 \\
64.5 \\
56.0 \\
48.0 \\
42.0 \\
36.5 \\
32.0 \\
28.0 \\
24.5\end{array}$ \\
\hline
\end{tabular}

* Use curve A then evapotranspiration losses are average or normal. Use curve B during excessively hot, windy veather. 
Table 1. Plotting data for base-flow recession curves - continued

\begin{tabular}{|c|c|c|c|c|c|c|c|c|c|c|}
\hline \multirow{4}{*}{$\begin{array}{l}\text { Time } \\
\text { in } \\
\text { days }\end{array}$} & \multicolumn{10}{|c|}{ Gaging station number and name, drainage area, and discharge in cfs for curves $A$ and $B *$} \\
\hline & \multirow{2}{*}{\multicolumn{2}{|c|}{$\begin{array}{l}\begin{array}{c}5-4220 \\
\text { Vapsipinicon River } \\
\text { near De Hitt }\end{array} \\
2330 \mathrm{sq} \mathrm{mi}\end{array}$}} & \multirow{2}{*}{\multicolumn{2}{|c|}{ 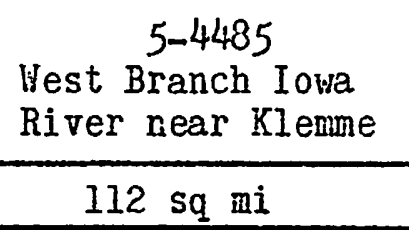 }} & \multirow{2}{*}{\multicolumn{2}{|c|}{$\begin{array}{l}\quad 5-4490 \\
\text { East Branch Iova } \\
\text { River near Klemme } \\
133 \mathrm{sq} \mathrm{mi}\end{array}$}} & \multirow{2}{*}{\multicolumn{2}{|c|}{$\begin{array}{c}5-4495 \\
\text { Iowa River } \\
\text { near Rowan } \\
429 \mathrm{sq} \mathrm{mi} \\
\end{array}$}} & \multicolumn{2}{|c|}{$\begin{array}{l}5-4515 \\
\text { Iowa River near } \\
\text { Harshalltown }\end{array}$} \\
\hline & & & & & & & & & $1564 \mathrm{~s}$ & ini \\
\hline & A & B & A & B & A & B & A & B & $\Lambda$ & B \\
\hline
\end{tabular}

* Use curve A when erapotranspiration losses are average or normal. Use curve $B$ during excessively hot, windy weather. 
Table 1. Plotting data for base-flow recession curves -- continued

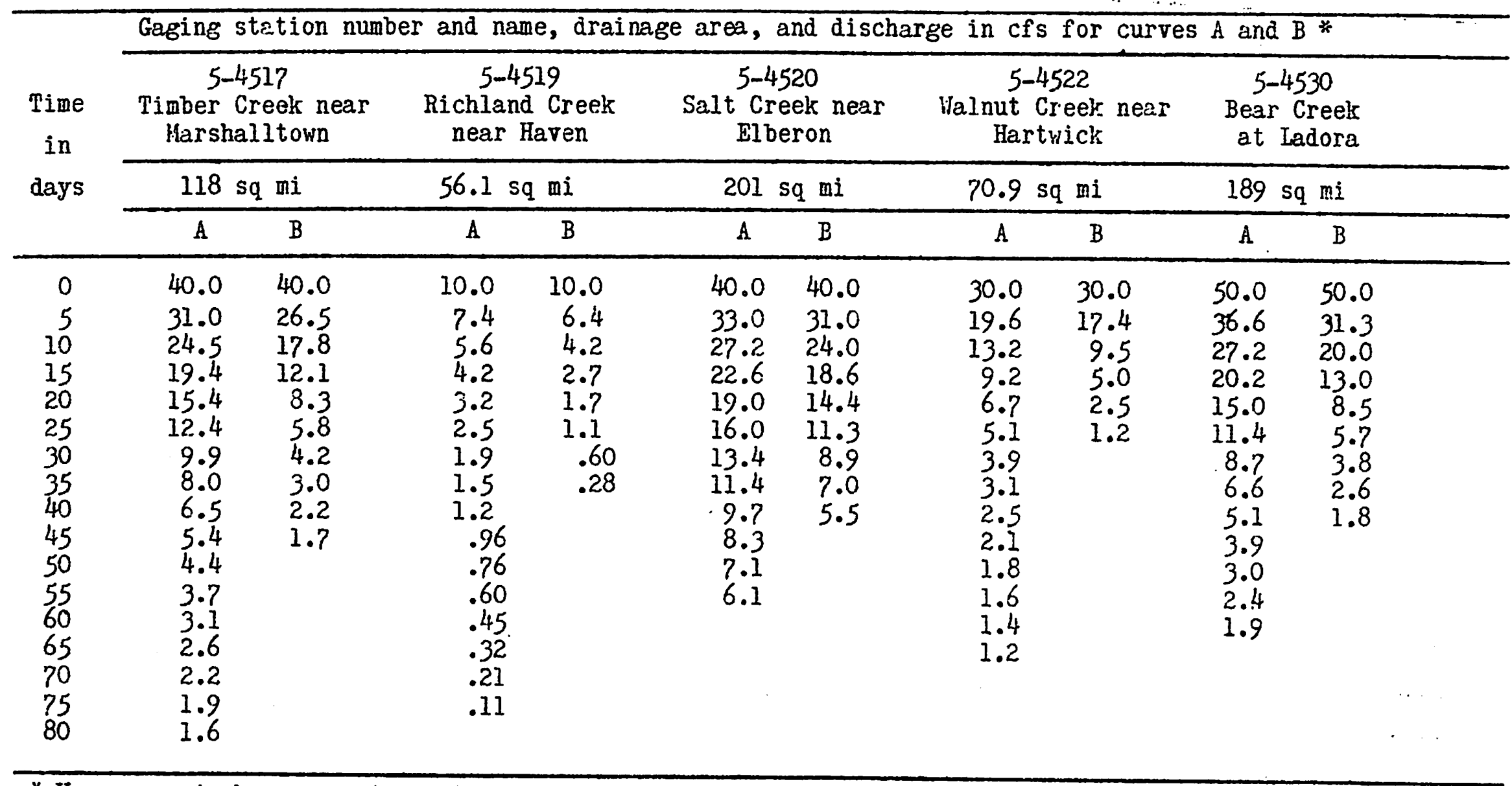

* Use curve A when evapotranspiration losses are average or normal. Use curve B during excessively hot, windy weather. 
Table 1. Plotting data for base-flow recession curves -- continued

\begin{tabular}{|c|c|c|c|c|c|c|c|c|c|c|}
\hline \multirow{4}{*}{$\begin{array}{l}\text { Time } \\
\text { in } \\
\text { days }\end{array}$} & \multicolumn{10}{|c|}{ Gaging station number and name, drainage area, and discharge in cfs for curves $A$ and $B *$} \\
\hline & \multicolumn{2}{|c|}{$\begin{array}{l}5-4531 \\
\text { Iowa River at } \\
\text { Marengo }\end{array}$} & \multicolumn{2}{|c|}{$\begin{array}{l}5-4540 \\
\text { Rapid Creeir near } \\
\text { Iowa City }\end{array}$} & \multicolumn{2}{|c|}{$\begin{array}{c}5-4550 \\
\text { Clear Creek near } \\
\text { Coralville }\end{array}$} & \multicolumn{2}{|c|}{$\begin{array}{l}5-4550 \\
\text { Ralston Creek } \\
\text { at Iowa City }\end{array}$} & \multicolumn{2}{|c|}{$\begin{array}{l}5-4555 \\
\text { english River } \\
\text { at Kalona }\end{array}$} \\
\hline & \multicolumn{2}{|c|}{$279 \div \mathrm{sq} \mathrm{mi}$} & \multicolumn{2}{|c|}{$24.6 \mathrm{sq} \mathrm{mi}$} & \multicolumn{2}{|c|}{$98.1 \mathrm{sq} \mathrm{mi}$} & \multicolumn{2}{|c|}{$3.01 \mathrm{sq} \mathrm{mi}$} & \multicolumn{2}{|c|}{$573 \mathrm{sq} \mathrm{mi}$} \\
\hline & A & B & $\dot{i}$ & B & A & B & A & B & A & B \\
\hline $\begin{array}{r}0 \\
5 \\
10 \\
15 \\
20 \\
25 \\
30 \\
35 \\
40 \\
45 \\
50 \\
55 \\
60 \\
65 \\
70 \\
75 \\
80 \\
85 \\
90 \\
95 \\
100\end{array}$ & $\begin{array}{r}1000 \\
790 \\
640 \\
i 520 \\
425 \\
350 \\
294 \\
250 \\
213 \\
183 \\
159 \\
135 \\
122\end{array}$ & $\begin{array}{r}1000 \\
745 \\
565 \\
435 \\
344 \\
272 \\
222 \\
184 \\
154 \\
130 \\
111\end{array}$ & $\begin{array}{r}3.0 .0 \\
6.2 \\
3.9 \\
2.5 \\
1.5 \\
.93 \\
.51 \\
.20\end{array}$ & $\begin{array}{c}10.0 \\
6.0 \\
3.2 \\
1.6 \\
.69 \\
.26\end{array}$ & $\begin{array}{r}60.0 \\
41.0 \\
27.0 \\
18.1 \\
12.0 \\
8.0 \\
5.6 \\
4.0 \\
2.9 \\
2.2 \\
1.8\end{array}$ & $\begin{array}{r}60.0 \\
41.0 \\
26.0 \\
16.0 \\
9.4 \\
5.5 \\
3.4 \\
2.2 \\
1.4\end{array}$ & $\begin{array}{l}1.9 \\
1.0 \\
.51 \\
.24 \\
.10 \\
.044\end{array}$ & $\begin{array}{c}1.9 \\
.82 \\
.31 \\
.11 \\
.032\end{array}$ & $\begin{array}{r}200 \\
138 \\
99.0 \\
71.5 \\
53.0 \\
39.5 \\
30.0 \\
23.2 \\
18.4 \\
14.7 \\
11.8 \\
9.6 \\
7.9 \\
6.6 \\
5.0 \\
4.8 \\
4.1 \\
3.5 \\
3.2 \\
2.3 \\
2.5\end{array}$ & $\begin{array}{r}200 \\
112 \\
660 \\
40.5 \\
25.0 \\
16.0 \\
10.4 \\
6.9 \\
4.8 \\
3.4 \\
2.4\end{array}$ \\
\hline
\end{tabular}

* Use curve A when evapotranspiration losses are arerage or normal:Use curve B during excessively hot, windy weather. 
Table 1. Plotting data for base-flow recession curves -- continued

\begin{tabular}{|c|c|c|c|c|c|c|c|c|c|c|}
\hline \multirow{3}{*}{$\begin{array}{l}\text { Tine } \\
\text { in } \\
\text { days }\end{array}$} & \multicolumn{4}{|c|}{$\begin{array}{cc}5-4580 & 5-4585 \\
\text { Iittle Cedar River } & \text { Cedar River at } \\
\text { near Ionia } & \text { Janesville }\end{array}$} & \multicolumn{2}{|c|}{$\begin{array}{l}5-4589 \\
\text { West Fork Cedar River } \\
\text { at Finchford }\end{array}$} & \multicolumn{2}{|c|}{$\begin{array}{l}\quad 5-4590 \\
\text { Shel1 Rock River } \\
\text { near Northwood }\end{array}$} & \multicolumn{2}{|c|}{$\begin{array}{c}5-4595 \\
\text { linnebago River } \\
\text { at liason City }\end{array}$} \\
\hline & \multicolumn{2}{|c|}{$306 \mathrm{sq} \mathrm{mi}$} & \multicolumn{2}{|c|}{$1661 \mathrm{sq} \mathrm{mi}$} & \multicolumn{2}{|c|}{$846 \mathrm{sq} \mathrm{mi}$} & \multicolumn{2}{|c|}{$300 \mathrm{sq} \mathrm{mi}$} & \multicolumn{2}{|c|}{$526 \mathrm{sq} \mathrm{mi}$} \\
\hline & $A$ & B & A & $B$ & A & $\mathrm{B}$ & $\dot{A}$ & $B$ & $A$ & B \\
\hline
\end{tabular}

* Use curve $A$ when evapotranspiration losses are average or normal. Use curve $B$ during excessively hot, windy weather. 
Table 1. Plotting data for base-flow recession curves -- continued

\begin{tabular}{|c|c|c|c|c|c|c|c|c|c|c|}
\hline \multirow{4}{*}{$\begin{array}{l}\text { Tine } \\
\text { in } \\
\text { days }\end{array}$} & \multicolumn{10}{|c|}{ Gaging station nunber and name, drainage area, and discharge in cfs for curves $A$ and $B *$} \\
\hline & \multicolumn{2}{|c|}{$\begin{array}{c}5-4620 \\
\text { Shell Rocik River } \\
\text { at Shell Rock }\end{array}$} & \multicolumn{2}{|c|}{$\begin{array}{c}5-4630 \\
\text { Beaver Creek at } \\
\text { New Hartford }\end{array}$} & \multicolumn{2}{|c|}{$\begin{array}{c}5-4635 \\
\text { Blackhawk Creek } \\
\text { at Hudson }\end{array}$} & \multicolumn{2}{|c|}{$\begin{array}{l}5-4640 \\
\text { Cedar River } \\
\text { at Waterloo }\end{array}$} & \multicolumn{2}{|c|}{$\begin{array}{l}5-4645 \\
\text { Cedar River at } \\
\text { Cedar Rapids }\end{array}$} \\
\hline & \multicolumn{2}{|c|}{$1746 \mathrm{sq} \mathrm{mi}$} & \multicolumn{2}{|c|}{$347 \mathrm{sq} \mathrm{mi}$} & \multicolumn{2}{|c|}{$303 \mathrm{sq} \mathrm{mi}$} & \multicolumn{2}{|c|}{$5146 \mathrm{sq} \mathrm{mi}$} & \multicolumn{2}{|c|}{$6510 \mathrm{sq} \mathrm{mi}$} \\
\hline & A & B & A & $\bar{B}$ & A & $\bar{B}$ & $\bar{A}$ & $\vec{B}$ & A & $\bar{B}$ \\
\hline $\begin{array}{l}0 \\
5 \\
10 \\
15 \\
20 \\
25 \\
30 \\
35 \\
40 \\
45 \\
50 \\
55 \\
60 \\
65 \\
70 \\
75 \\
80\end{array}$ & $\begin{array}{r}1400 \\
1120 \\
905 \\
745 \\
615 \\
515 \\
435 \\
374 \\
324 \\
282 \\
248 \\
222 \\
198 \\
178 \\
162 \\
148 \\
136\end{array}$ & $\begin{array}{r}1400 \\
1040 \\
800 \\
622 \\
490 \\
390 \\
316 \\
260 \\
215 \\
181 \\
153 \\
131 \\
113\end{array}$ & $\begin{array}{r}100 \\
71.0 \\
52.0 \\
39.4 \\
30.0 \\
23.4 \\
18.4 \\
14.7 \\
12.0 \\
9.9 \\
8.3 \\
7.0 \\
6.0 \\
5.2 \\
4.5\end{array}$ & $\begin{array}{r}100 \\
61.5 \\
39.5 \\
26.0 \\
17.9 \\
12.7 \\
9.4 \\
7.0 \\
5.4 \\
4.3\end{array}$ & $\begin{array}{r}100 \\
56.5 \\
35.5 \\
24.2 \\
17.3 \\
12.8 \\
9.6 \\
7.2 \\
5.2\end{array}$ & $\begin{array}{r}100 \\
41.0 \\
20.5 \\
11.3 \\
6.8 \\
4.1\end{array}$ & $\begin{array}{r}3000 \\
2560 \\
2220 \\
1930 \\
1680 \\
1470 \\
1300 \\
1160 \\
1030 \\
925 \\
840 \\
760 \\
695 \\
640 \\
590 \\
550 \\
510\end{array}$ & $\begin{array}{r}3000 \\
2420 \\
2020 \\
1690 \\
1440 \\
1230 \\
1060 \\
935 \\
835 \\
745 \\
670 \\
605 \\
550\end{array}$ & $\begin{array}{r}7000 \\
5600 \\
4480 \\
3620 \\
2980 \\
2450 \\
2050 \\
1730 \\
1490 \\
1260 \\
1080 \\
950 \\
840\end{array}$ & $\begin{array}{l}7000 \\
5400 \\
4150 \\
3220 \\
2500 \\
1960 \\
1540 \\
1230 \\
980 \\
795 \\
640\end{array}$ \\
\hline
\end{tabular}

* Use curve A when evapotranspiration losses are average or normal. Use curve $B$ during excessively hot, windy weather. 
Table 1. Plotting data for base-flow recession curves - continued

\begin{tabular}{|c|c|c|c|c|c|c|c|c|c|c|c|}
\hline \multirow{4}{*}{$\begin{array}{l}\text { Time } \\
\text { in } \\
\text { days }\end{array}$} & \multicolumn{11}{|c|}{ Gaging station number and name, drainage area, and discharge in cís for curves $A$ and $B *$} \\
\hline & \multicolumn{2}{|c|}{$\begin{array}{l}5-4650 \\
\text { Cedar River near } \\
\text { Conesville }\end{array}$} & \multicolumn{2}{|c|}{$\begin{array}{l}5-4700 \\
\text { Skunk River } \\
\text { near Ames. }\end{array}$} & \multicolumn{2}{|c|}{$\begin{array}{l}5-4705 \\
\text { Squaw Creek } \\
\text { at Ames }\end{array}$} & \multicolumn{2}{|c|}{$\begin{array}{c}5-4710 \\
\text { Skunk River below Squaw } \\
\text { Creek near Ames.. }\end{array}$} & \multicolumn{3}{|c|}{$\begin{array}{l}5-4712 \\
\text { Indian Creeli } \\
\text { near Hingo }\end{array}$} \\
\hline & \multicolumn{2}{|c|}{$7785 \mathrm{sq} \mathrm{mi}$} & \multicolumn{2}{|c|}{$315 \mathrm{sq} \mathrm{mi}$} & \multicolumn{2}{|c|}{$204 \mathrm{sq} \mathrm{mi}$} & \multicolumn{2}{|c|}{$556 \mathrm{sq} \mathrm{mi}$} & \multicolumn{2}{|c|}{$276 \mathrm{sq} \mathrm{mi}$} & \\
\hline & A & $\bar{B}$ & A & B & $\bar{A}$ & $\bar{B}$ & A & $B$ & $A$ & $B$ & \\
\hline $\begin{array}{l}0 \\
5 \\
10 \\
15 \\
20 \\
25 \\
30 \\
35 \\
40 \\
45 \\
50 \\
55 \\
60 \\
65 \\
70 \\
75 \\
80 \\
85 \\
90\end{array}$ & $\begin{array}{r}10,000 \\
7,700 \\
6,050 \\
4,850 \\
3,900 \\
3,180 \\
2,640 \\
2,180 \\
1,850 \\
1,590 \\
1,370 \\
1,200 \\
1,060 \\
940 \\
845 \\
760 \\
695 \\
635 \\
585\end{array}$ & $\begin{array}{r}10,000 \\
7,000 \\
5,040 \\
3,720 \\
2,800 \\
2,160 \\
1,700 \\
1,360 \\
1,120 \\
940 \\
800\end{array}$ & $\begin{array}{r}100 \\
65.0 \\
42.5 \\
27.5 \\
18.0 \\
11.8 \\
7.8 \\
5.2 \\
3.4\end{array}$ & $\begin{array}{r}100 \\
54.0 \\
28.5 \\
15.0 \\
7.7 \\
3.9\end{array}$ & $\begin{array}{r}100 \\
54.5 \\
30.0 \\
16.4 \\
8.8 \\
4.7 \\
2.5\end{array}$ & $\begin{array}{r}100 \\
47.5 \\
20.5 \\
3.4 \\
2.9\end{array}$ & $\begin{array}{r}190 \\
140 \\
102 \\
74.0 \\
53.0 \\
37.5 \\
26.5 \\
18.5 \\
13.0 \\
9.1 \\
6.3\end{array}$ & $\begin{array}{r}190 \\
116 \\
70.0 \\
42.0 \\
24.5 \\
14.0 \\
7.7\end{array}$ & $\begin{array}{r}100 \\
67.5 \\
47.0 \\
32.5 \\
22.8 \\
16.2 \\
11.7 \\
8.5 \\
6.3 \\
4.7\end{array}$ & $\begin{array}{r}100 \\
51.0 \\
27.3 \\
14.8 \\
8.3 \\
4.6\end{array}$ & \\
\hline
\end{tabular}

* Use curve A when errpotranspiration losses are average or normal. Use curve $B$ during excessively hot, windy weather. 
Table 1. Plotting data for base-flow recession curves -- continued

\begin{tabular}{|c|c|c|c|c|c|c|c|c|c|c|c|}
\hline \multirow{3}{*}{$\begin{array}{l}\text { Time } \\
\text { in } \\
\text { days }\end{array}$} & \multicolumn{2}{|c|}{$\begin{array}{l}5-4715 \\
\text { Sirunk River near } \\
\text { Oskaloosa }\end{array}$} & \multicolumn{3}{|c|}{$\begin{array}{l}5-4725 \\
\text { iyorth Skunir River } \\
\text { near. Sïgourney: }\end{array}$} & \multicolumn{2}{|c|}{$\begin{array}{l}\quad 5-4735 \\
\text { Bis Creek near } \\
\text { liount Pleasant }\end{array}$} & \multicolumn{2}{|c|}{$\begin{array}{l}5-4740 \\
\text { Sirunk River } \\
\text { at Augusta }\end{array}$} & \multicolumn{2}{|c|}{$\begin{array}{l}5-4765 \\
\text { Vest Fork Des lioinas } \\
\text { River at Esthervili }\end{array}$} \\
\hline & \multicolumn{2}{|c|}{$1635 \mathrm{sq} \mathrm{mi}$} & \multicolumn{3}{|c|}{$730 \mathrm{sq} \mathrm{mi}$} & \multicolumn{2}{|c|}{$106 \mathrm{sq} \mathrm{\textrm { } \mathrm { i }}$} & \multicolumn{2}{|c|}{$4303 \mathrm{sa} \mathrm{mi}$} & \multicolumn{2}{|c|}{$1372 \mathrm{sq} \mathrm{mi}$} \\
\hline & is & $B$ & & A & B & $\dot{A}$ & $B$ & $A$ & $\bar{B}$ & $\dot{A}$ & $\bar{B}$ \\
\hline
\end{tabular}

* Use curve A when evapotranspiration losses are average or normal. Use curve $B$ durinf excessively hot, windy weather. 
Table 1. Plotting data for base-flow recession curves -- continued

\begin{tabular}{|c|c|c|c|c|c|c|c|c|c|c|c|}
\hline \multirow{4}{*}{$\begin{array}{l}\text { Time } \\
\text { in } \\
\text { days }\end{array}$} & \multicolumn{11}{|c|}{ Gaging station number and name, drainage area, and discharge in cfs for curves $A$ and $B^{*}$} \\
\hline & \multicolumn{3}{|c|}{$\begin{array}{l}\quad 5-4780 \\
\text { East Fork Des Koines } \\
\text { River near Burt. }\end{array}$} & \multicolumn{2}{|c|}{$\begin{array}{l}5-4790 \\
\text { East Fork Des lioines } \\
\text { River at Dakota City }\end{array}$} & \multicolumn{2}{|c|}{$\begin{array}{l}5-4800 \\
\text { Lizard Creek } \\
\text { near Clare }\end{array}$} & \multicolumn{2}{|c|}{$\begin{aligned} & 5-4805 \\
& \text { Des Moines River } \\
& \text { at Fort Dodge }\end{aligned}$} & \multicolumn{2}{|c|}{$\begin{array}{l}5-4810 \\
\text { Boone River nea } \\
\text { Wubster City }\end{array}$} \\
\hline & \multicolumn{3}{|c|}{$462 \mathrm{sq} \mathrm{mi}$} & \multicolumn{2}{|c|}{$1308 \mathrm{sq} \mathrm{mi}$} & \multicolumn{2}{|c|}{$25 ? \mathrm{sq} \mathrm{mi}$} & \multicolumn{2}{|c|}{$4190 \mathrm{sq} \mathrm{mi}$} & \multicolumn{2}{|c|}{$844 \mathrm{sq} \mathrm{mi}$} \\
\hline & A & $B$ & & A & $B$ & A & $B$ & A & . B & A & B \\
\hline $\begin{array}{l}0 \\
5 \\
10 \\
15 \\
20 \\
25 \\
30 \\
35 \\
40 \\
45 \\
50 \\
55 \\
60\end{array}$ & $\begin{array}{r}60.0 \\
44.0 \\
28.6 \\
16.4 \\
9.1 \\
5.7 \\
3.7 \\
2.7 \\
2.0\end{array}$ & $\begin{array}{r}60.0 \\
37.0 \\
19.3 \\
9.4 \\
5.0 \\
3.0 \\
2.1\end{array}$ & & $\begin{array}{l}500 \\
348 \\
250 \\
185 \\
138 \\
105 \\
80.0 \\
62.5 \\
50.0 \\
39.6 \\
32.2 \\
26.3 \\
21.8 \\
18.5 \\
16.0\end{array}$ & $\begin{array}{l}500 \\
310 \\
200 \\
133 \\
91.0 \\
65.0 \\
47.5 \\
35.4 \\
26.8 \\
21.0 \\
17.0\end{array}$ & $\begin{array}{r}140 \\
108 \\
85.8 \\
67.0 \\
53.0 \\
42.0 \\
33.4 \\
26.4 \\
21.0 \\
16.7 \\
13.3 \\
10.4 \\
8.3 \\
6.6 \\
5.3 \\
4.2 \\
3.3\end{array}$ & $\begin{array}{l}140 \\
100 \\
72.0 \\
52.5 \\
38.5 \\
28.4 \\
21.2 \\
15.8 \\
12.0 \\
9.1 \\
7.0 \\
5.3 \\
4.1\end{array}$ & $\begin{array}{c}1400 \\
1060 \\
820 \\
625 \\
485 \\
377 \\
300 \\
243 \\
198 \\
162 \\
135 \\
114 \\
98.0\end{array}$ & $\begin{array}{r}1400 \\
945 \\
640 \\
445 \\
319 \\
232 \\
175 \\
134 \\
106\end{array}$ & $\begin{array}{r}230 \\
148 \\
98.0 \\
67.0 \\
46.5 \\
33.4 \\
25.0 \\
19.0 \\
14.8 \\
11.8 \\
9.4\end{array}$ & $\begin{array}{r}230 \\
138 \\
86.0 \\
54.5 \\
35.6 \\
24.0 \\
17.0 \\
12.0 \\
9.0\end{array}$ \\
\hline
\end{tabular}

* Use curve A when evapotranspiration losses are average or normal.

Use curve $B$ during excessively hot, windy weather. 
Table 1. Plotting data for base-flow recession curves -- continued

\begin{tabular}{|c|c|c|c|c|c|c|c|c|c|c|}
\hline \multirow{4}{*}{$\begin{array}{l}\text { Time } \\
\text { in } \\
\text { days }\end{array}$} & \multicolumn{10}{|c|}{ Gaging station number and name, drainage area, and discharge in cfs for curves $A$ and $B *$} \\
\hline & \multicolumn{2}{|c|}{$\begin{array}{l}5-4815 \\
\text { Des Moines River } \\
\text { rear Boone }\end{array}$} & \multicolumn{2}{|c|}{$\begin{array}{l}5-4821.7 \\
\text { Big Cedar Creek } \\
\text { near Varina }\end{array}$} & \multicolumn{2}{|c|}{$\begin{array}{l}5-4823 \\
\text { North Raccoon River } \\
\text { near Sac City }\end{array}$} & \multicolumn{2}{|c|}{$\begin{array}{c}5-4825 \\
\text { North Raccoon River } \\
\text { near Jefferson }\end{array}$} & \multicolumn{2}{|c|}{$\begin{array}{l}5-4830 \\
\text { inst Fork Hardin } \\
\text { Creek near Churdan }\end{array}$} \\
\hline & \multicolumn{2}{|c|}{ 5511. sq $\mathrm{mi}$} & \multicolumn{2}{|c|}{$80.0 \mathrm{sq} \mathrm{mi}$} & \multicolumn{2}{|c|}{$713 \mathrm{sqmi}$} & \multicolumn{2}{|c|}{$1519 \mathrm{sq} \mathrm{mi}$} & \multicolumn{2}{|c|}{$24.0 \mathrm{sq} \mathrm{mi}$} \\
\hline & $\bar{A}$ & $\bar{B}$ & $\overline{\mathrm{A}}$ & $\bar{B}$ & $\bar{A}$ & B & A. & $\bar{B}$ & $\dot{i}$ & $\overline{\mathrm{B}}$ \\
\hline $\begin{array}{l}0 \\
5 \\
10 \\
15 \\
20 \\
25 \\
30 \\
35 \\
40 \\
45 \\
50 \\
55 \\
60 \\
65 \\
70\end{array}$ & $\begin{array}{r}1500 \\
1110 \\
830 \\
630 \\
490 \\
390 \\
315 \\
256 \\
214 \\
182 \\
156 \\
134 \\
117\end{array}$ & $\begin{array}{r}1500 \\
1030 \\
720 \\
515 \\
380 \\
282 \\
215 \\
166 \\
134\end{array}$ & $\begin{array}{r}25.0 \\
16.0 \\
10.8 \\
7.6 \\
5.5 \\
4.1 \\
3.2 \\
2.5 \\
2.0 \\
1.6 \\
1.4 \\
1.2 \\
1.0\end{array}$ & $\begin{array}{r}25.0 \\
15.2 \\
9.3 \\
5.6 \\
3.4 \\
2.0 \\
1.2\end{array}$ & $\begin{array}{l}200 \\
136 \\
93.5 \\
66.0 \\
47.5 \\
35.0 \\
27.0 \\
21.0 \\
16.6 \\
13.2 \\
10.8\end{array}$ & $\begin{array}{r}200 \\
116 \\
71.0 \\
44.5 \\
30.0 \\
21.0 \\
15.2 \\
11.2 \\
8.4\end{array}$ & $\begin{array}{l}300 \\
222 \\
169 \\
129 \\
102 \\
80.5 \\
65.5 \\
54.0 \\
45.0 \\
38.0 \\
32.6 \\
28.5 \\
25.4 \\
22 . \\
20.0\end{array}$ & $\begin{array}{l}300 \\
200 \\
139 \\
100 \\
74.0 \\
55.0 \\
42.5 \\
33.8 \\
28.0\end{array}$ & $\begin{array}{r}10.0 \\
6.2 \\
3.8 \\
2.3 \\
1.4 \\
.82 \\
.48 \\
.27 \\
.15\end{array}$ & $\begin{array}{r}10.0 \\
4.8 \\
2.3 \\
1.2 \\
. .43 \\
.16\end{array}$ \\
\hline
\end{tabular}

* Use curve A vhen evapctranspiration losses are average or normal. Use curve B during excessively hot, windy weather. 
Table 1. Plotting data for base-flow recession curves -- continued

\begin{tabular}{|c|c|c|c|c|c|c|c|c|c|c|}
\hline \multirow{3}{*}{$\begin{array}{l}\text { Time } \\
\text { in } \\
\text { days }\end{array}$} & \multicolumn{2}{|c|}{$\begin{array}{c}5-4836 \\
\text { Hiddle Raccoon River } \\
\text { a.t Panora }\end{array}$} & \multicolumn{2}{|c|}{$\begin{array}{c}5-4840 \\
\text { South Raccoon River } \\
\text { at Redfield }\end{array}$} & \multicolumn{2}{|c|}{$\begin{array}{l}5-4845 \\
\text { Raccoon Rlver } \\
\text { at Van Heter }\end{array}$} & \multicolumn{2}{|c|}{$\begin{array}{c}5-4855 \\
\text { Des lioines River below } \\
\text { Raccoon at Des Hoines }\end{array}$} & \multicolumn{2}{|c|}{$\begin{array}{l}5-4860 \\
\text { North River } \\
\text { near Norwalk }\end{array}$} \\
\hline & \multicolumn{2}{|c|}{$440 \mathrm{sq} \mathrm{mi}$} & \multicolumn{2}{|c|}{$988 \mathrm{sq} \mathrm{mi}$} & \multicolumn{2}{|c|}{$3441 \mathrm{sq} \mathrm{ml}$} & \multicolumn{2}{|c|}{$9879 \mathrm{sq} \mathrm{mi}$} & \multicolumn{2}{|c|}{$349 \mathrm{sq} \mathrm{mi}$} \\
\hline & $\bar{A}$ & $\mathrm{~B}$ & A & B & $A$ & $\bar{B}$ & $\bar{A}$ & $\bar{B}$ & $\bar{\Lambda}$ & $\bar{B}$ \\
\hline $\begin{array}{l}0 \\
5 \\
10 \\
15 \\
20 \\
25 \\
30 \\
35 \\
40 \\
45 \\
50 \\
55 \\
60 \\
65 \\
70 \\
75 \\
80\end{array}$ & $\begin{array}{r}200 \\
144 \\
107 \\
83.0 \\
65.5 \\
53.5 \\
44.5 \\
38.0 \\
33.0 \\
29.0 \\
26.0 \\
23.5\end{array}$ & $\begin{array}{l}200 \\
117 \\
70.0 \\
45.0 \\
30.5 \\
22.0\end{array}$ & $\begin{array}{l}500 \\
366 \\
275 \\
208 \\
160 \\
126 \\
100 \\
79.0 \\
63.5 \\
51.5 \\
42.0 \\
34.6 \\
29.0 \\
24.8 \\
21.5\end{array}$ & $\begin{array}{r}500 \\
362 \\
263 \\
194 \\
144 \\
107 \\
80.0 \\
60.0 \\
46.0 \\
35.0 \\
27.0\end{array}$ & $\begin{array}{l}900 \\
660 \\
485 \\
355 \\
260 \\
190 \\
138 \\
103 \\
73.5 \\
57.5 \\
43.5\end{array}$ & $\begin{array}{l}900 \\
640 \\
455 \\
325 \\
233 \\
167 \\
120 \\
87.5 \\
64.0\end{array}$ & $\begin{array}{r}4300 \\
3100 \\
2260 \\
1670 \\
1270 \\
980 \\
775 \\
625 \\
512 \\
425 \\
355 \\
300 \\
260 \\
227 \\
200 \\
177 \\
158\end{array}$ & $\begin{array}{r}4300 \\
2780 \\
1810 \\
1170 \\
790 \\
540 \\
385 \\
282 \\
214 \\
166 \\
133\end{array}$ & $\begin{array}{r}100 \\
63.0 \\
38.5 \\
22.7 \\
13.3 \\
7.5 \\
4.2 \\
2.3\end{array}$ & $\begin{array}{r}100 \\
58.0 \\
30.5 \\
14.4 \\
6.0 \\
2.2\end{array}$ \\
\hline
\end{tabular}

* Use curve A when evapotranspiration losses are average or normal. Use curve $B$ during excessively hot, windy weather. 
Table 1. Plotting data for base-flow recession curves -. continued

\begin{tabular}{|c|c|c|c|c|c|c|c|c|c|c|c|}
\hline \multirow{4}{*}{$\begin{array}{l}\text { Time } \\
\text { in } \\
\text { days }\end{array}$} & \multicolumn{10}{|c|}{ Gaging station number and name, drainage area; and discharge in cfs for curves $A$ and $B *$} & \\
\hline & \multirow{2}{*}{\multicolumn{2}{|c|}{$\begin{array}{r}5-4864.9 \\
\text { Hiddle River near } \\
\text { Indianola } \\
503 \mathrm{sq} \mathrm{mi} \\
\end{array}$}} & \multicolumn{2}{|c|}{$\begin{array}{c}5-4374.7 \\
\text { South River near } \\
\text { Ackworth }\end{array}$} & \multicolumn{2}{|c|}{$\begin{array}{c}5-4885 \\
\text { Des Hoines River } \\
\text { near Tracy }\end{array}$} & \multicolumn{2}{|c|}{$\begin{array}{l}5-4890 \\
\text { Cedar Creek } \\
\text { near Bussey }\end{array}$} & \multicolumn{2}{|c|}{$\begin{array}{l}5-4395 \\
\text { Des lioines River } \\
\text { at Ottuma }\end{array}$} & \\
\hline & & & $460 \mathrm{sc}$ & mi & 12,479 & $\mathrm{sq} \mathrm{mi}$ & $374 \mathrm{sc}$ & $\mathrm{mi}$ & 13,372 & $\mathrm{mi}$ & \\
\hline & $\Lambda$ & $B$ & A & $B$ & A & $B$ & $\Lambda$ & $B$ & A & $B$ & \\
\hline $\begin{array}{r}0 \\
5 \\
10 \\
15 \\
20 \\
25 \\
30 \\
35 \\
40 \\
45 \\
50 \\
55 \\
80 \\
65 \\
70 \\
75 \\
80 \\
85 \\
90 \\
95 \\
100\end{array}$ & $\begin{array}{r}100 \\
73.0 \\
54.0 \\
40.5 \\
30.0 \\
21 . ? \\
15.0 \\
9.9 \\
5 . ?\end{array}$ & $\begin{array}{r}100 \\
68.0 \\
43.5 \\
25.4 \\
13.0 \\
5.7\end{array}$ & $\begin{array}{r}100 \\
47.5 \\
24.2 \\
13.3 \\
7.8 \\
4.9 \\
3.2 \\
2.2 \\
1.6\end{array}$ & $\begin{array}{r}100 \\
41.0 \\
16.5 \\
6.6 \\
2.6 \\
1.0\end{array}$ & $\begin{array}{r}8000 \\
5950 \\
4600 \\
3550 \\
2730 \\
2200 \\
1760 \\
1410 \\
1150 \\
940 \\
730 \\
650 \\
550 \\
470 \\
405 \\
352 \\
310 \\
272 \\
240 \\
214 \\
190\end{array}$ & $\begin{array}{r}8000 \\
5550 \\
3900 \\
2780 \\
2010 \\
1480 \\
1110 \\
835 \\
640 \\
500 \\
390 \\
308 \\
250\end{array}$ & $\begin{array}{r}15.0 \\
10.2 \\
7.3 \\
5.3 \\
3.9 \\
3.0 \\
2.4 \\
1.9 \\
1.6 \\
1.3 \\
1.1 \\
.96 \\
.84\end{array}$ & $\begin{array}{r}15.0 \\
8.8 \\
5.5 \\
3.6 \\
2.5 \\
1.8 \\
1.3 \\
1.0 \\
.80\end{array}$ & $\begin{array}{r}8000 \\
5750 \\
4250 \\
3140 \\
2400 \\
1870 \\
1480 \\
1180 \\
960 \\
780 \\
650 \\
545 \\
465 \\
400 \\
345 \\
304 \\
270\end{array}$ & $\begin{array}{r}8000 \\
5400 \\
3720 \\
2600 \\
1850 \\
1360 \\
1010 \\
760 \\
580 \\
445 \\
350 \\
280 \\
228\end{array}$ & $\cdot$ \\
\hline
\end{tabular}

* Use curre A when evapotranspiration losses are average or normal. Use curye $B$ during excessively hot, windy weather. 
Table 1. Plotting data for base-flow recession curves -- continued

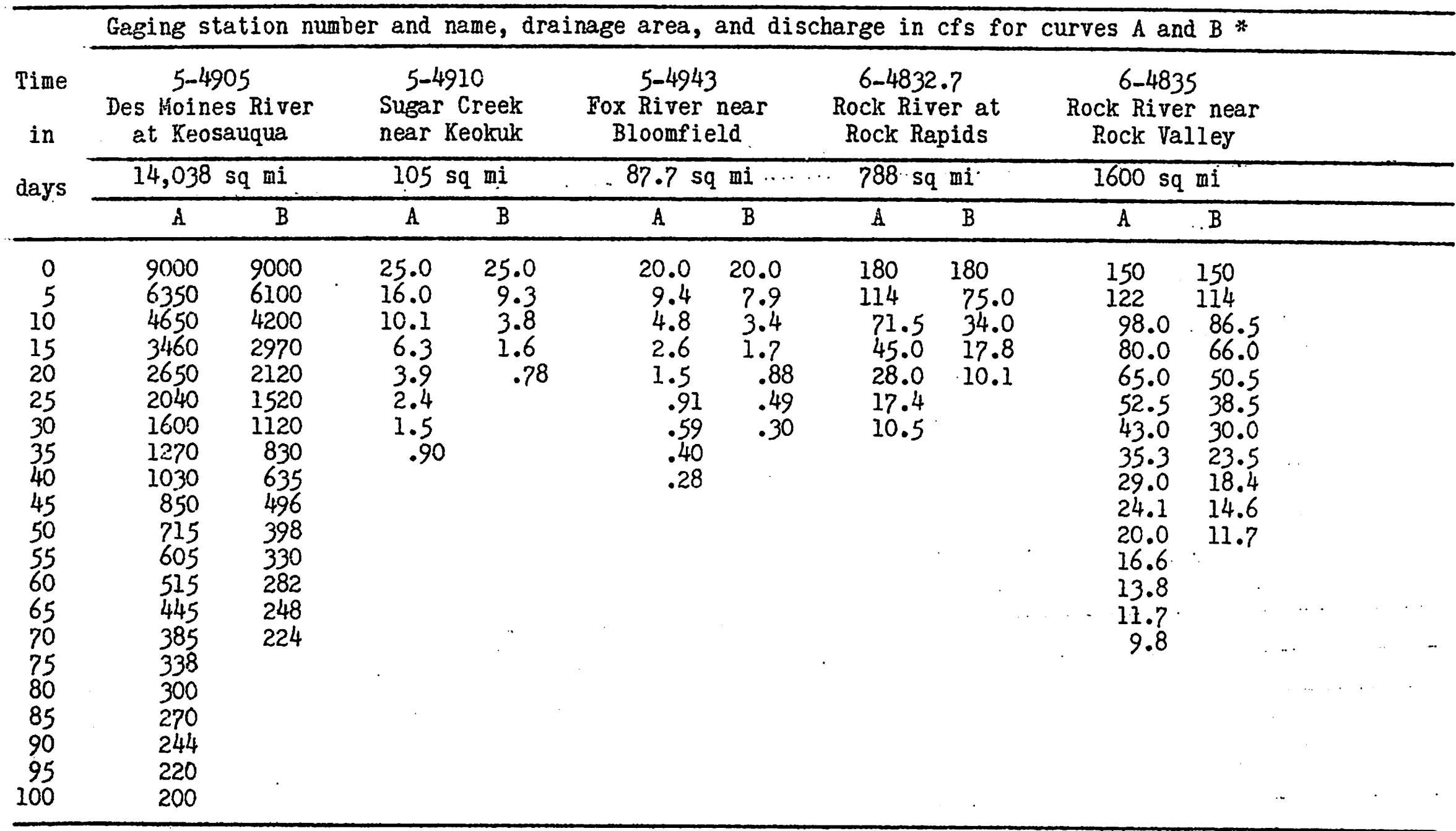

* Use curve A when evapotranspiration losses are average-or normal. Use curve B during excessively hot, windy weather. 
Table 1. Plotting data for base-flow recession curves -- continued

\begin{tabular}{|c|c|c|c|c|c|c|c|c|c|c|}
\hline \multirow{4}{*}{$\begin{array}{l}\text { Time } \\
\text { in } \\
\text { days }\end{array}$} & \multicolumn{10}{|c|}{ Gaging station number and name, drainage area, and discharge in cfs for curves $\dot{A}$ and $B^{*}$} \\
\hline & \multicolumn{2}{|c|}{$\begin{array}{l}6-4840 \\
\text { Dry Creek at } \\
\text { Hawarden }\end{array}$} & \multicolumn{2}{|c|}{$\begin{array}{l}\quad 6-6000 \\
\text { Perry Creek at 38th } \\
\text { Street Sioux City }\end{array}$} & \multicolumn{2}{|c|}{$\begin{array}{l}\text { 6-6001 } \\
\text { Floyd River } \\
\text { at Alton }\end{array}$} & \multicolumn{2}{|c|}{$\begin{array}{l}\quad 6-6003 \\
\text { Vest Branch Floyd } \\
\text { River near Struble }\end{array}$} & \multicolumn{2}{|c|}{$\begin{array}{l}6-6005 \\
\text { Floyd River } \\
\text { at James }\end{array}$} \\
\hline & \multicolumn{2}{|c|}{$48.4 \mathrm{sq}$ mi } & \multicolumn{2}{|c|}{$65.1 \mathrm{sq} \mathrm{mi}$} & \multicolumn{2}{|c|}{$265 \mathrm{sq} \mathrm{mi}$} & \multicolumn{2}{|c|}{$181 \mathrm{sq} \mathrm{mi}$} & \multicolumn{2}{|c|}{$882 \mathrm{sq} \mathrm{mi}$} \\
\hline & 1 & $\mathrm{~B}$ & A & B & $\dot{A}$ & B & A & B & A & $B$ \\
\hline
\end{tabular}

* Use curve A when evapotranspiration losses are average or normal. ঢse curve $B$ during excessively hot, windy weather. 
Table 1. Plotting data for base-flow recession curves - continued

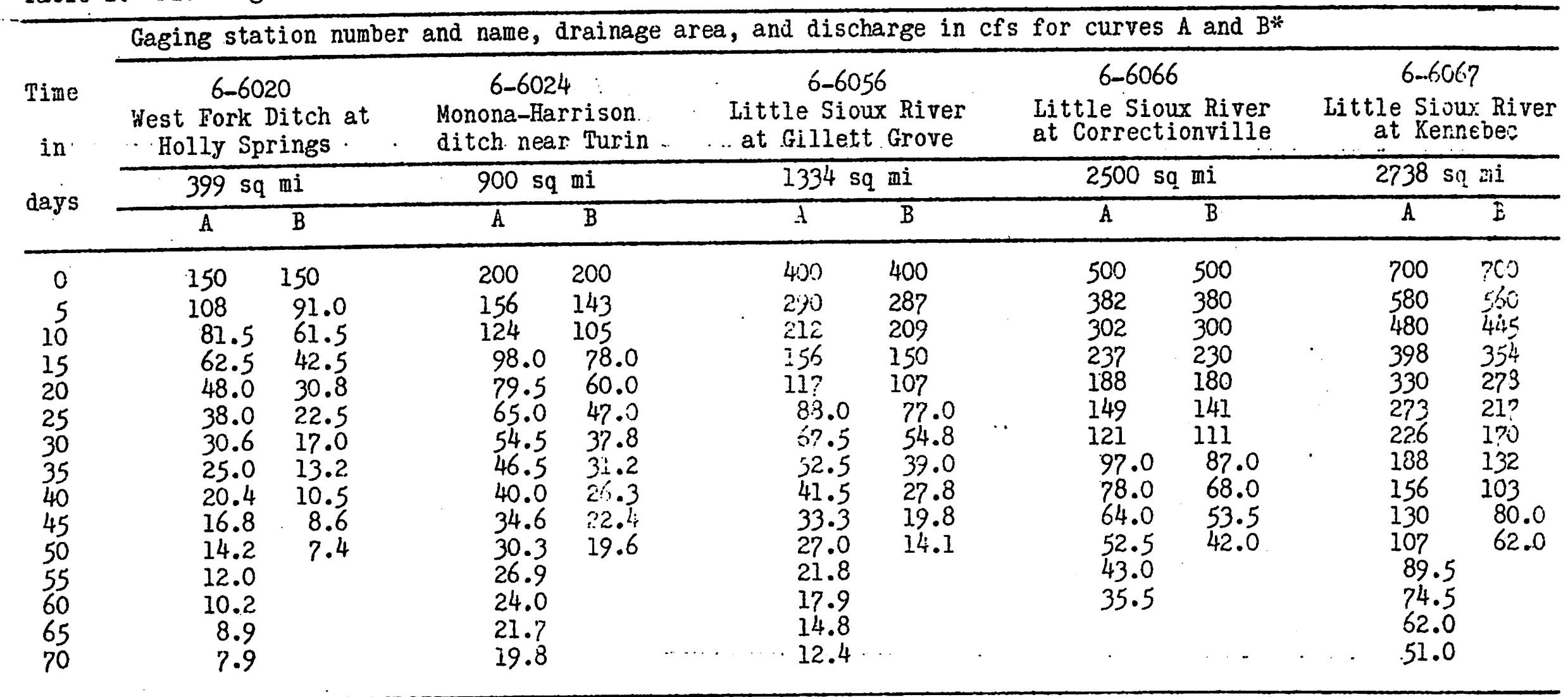

* Use curve A when evapotranspiration losses are average or normal. Use curve $B$ during excessively hot, windy weather. 
Table 1. Plotting data for base-flow recession curves -- continued

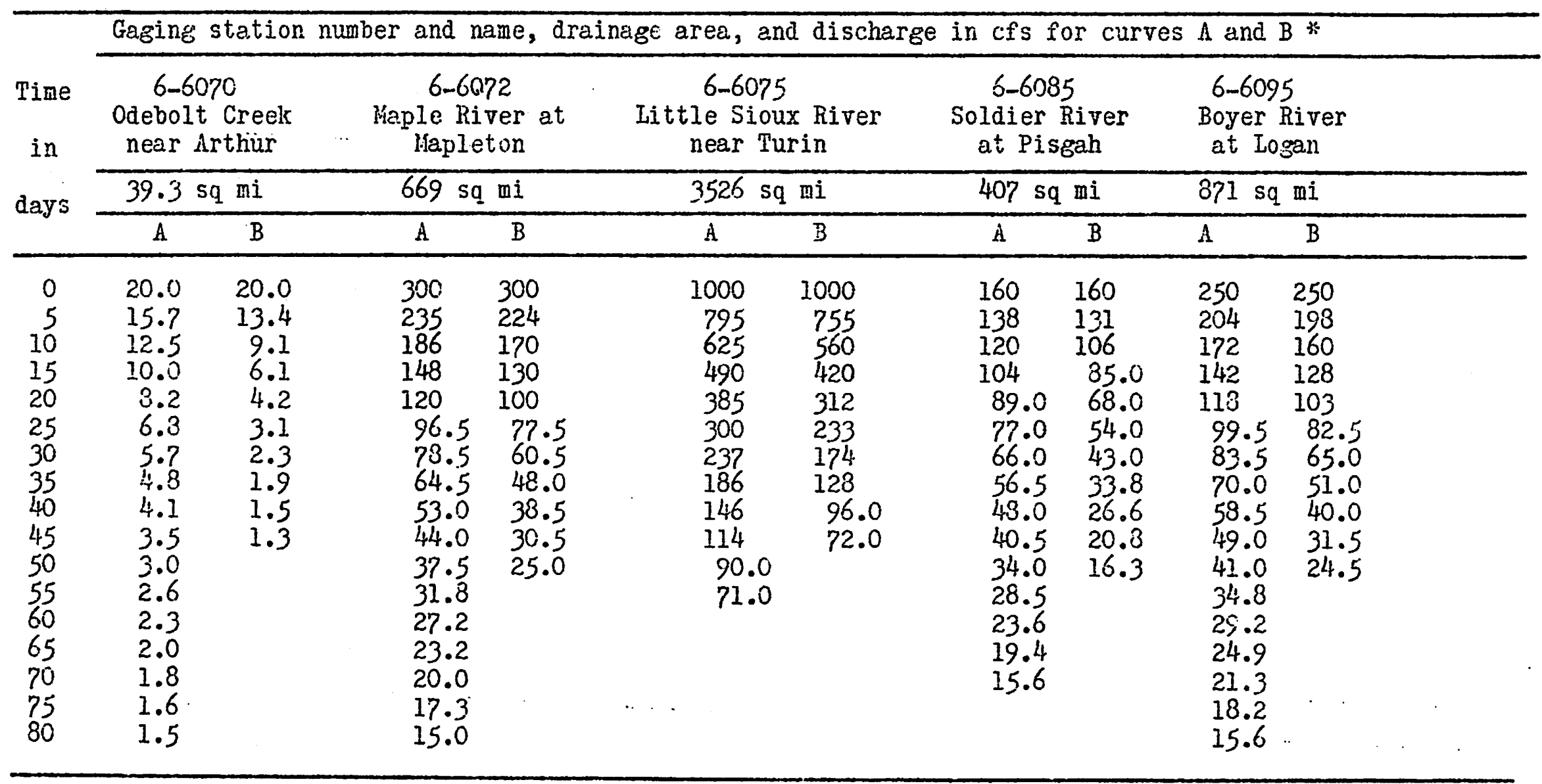

* Use curve A when evapotranspiration losses are average or normal. Use curve $B$ during excessively hot, windy weather. 
Table 1. Plotting data for base-flow recession curves -- continued

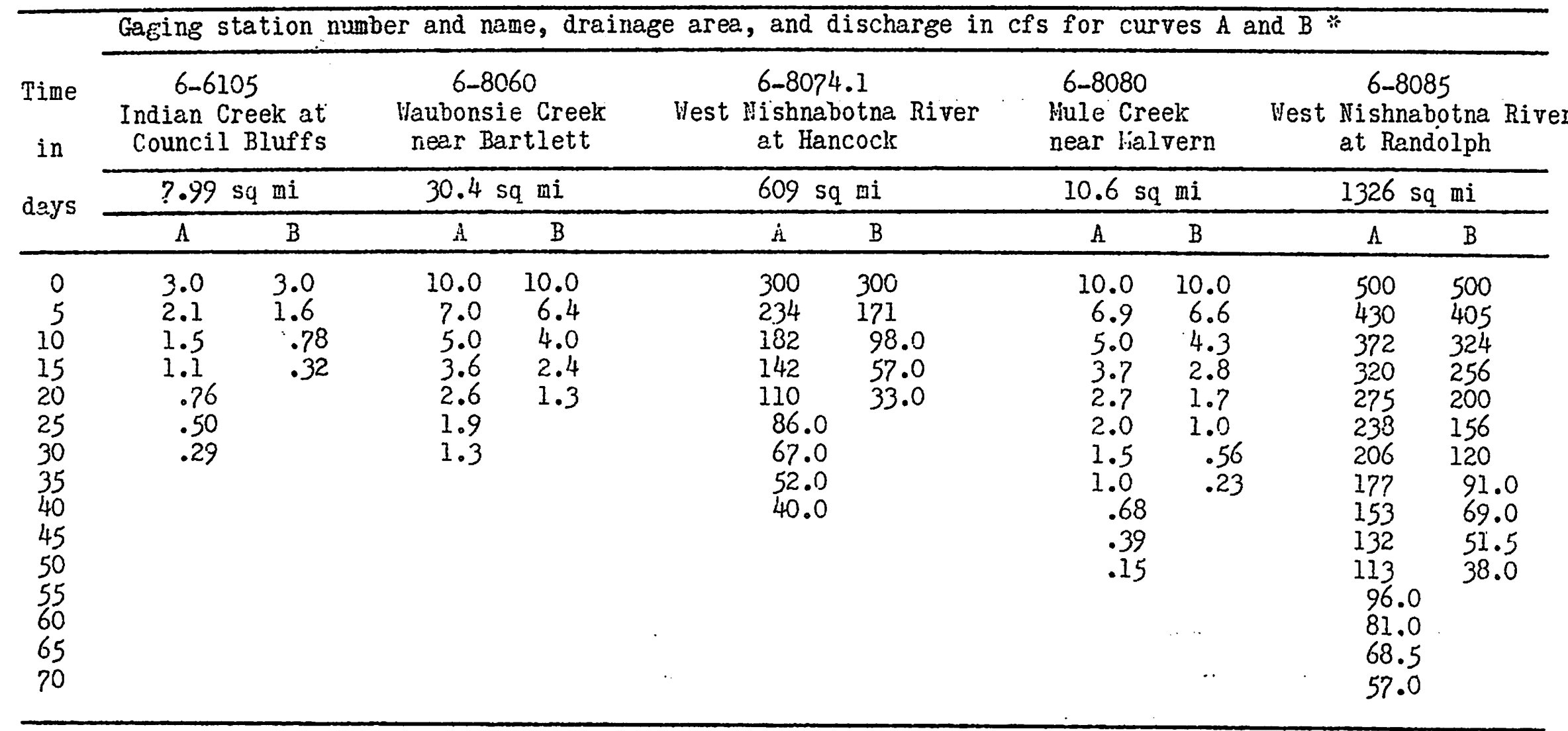

* Use curve A when evapotranspiration losses are average or normal. Use curve $B$ during excessively hot, windy weather. 
Table 1. Plotting data for base-flow recession curves - continued

\begin{tabular}{|c|c|c|c|c|c|c|c|c|c|c|c|}
\hline \multirow{3}{*}{$\begin{array}{l}\text { Time } \\
\text { in } \\
\text { days }\end{array}$} & \multicolumn{5}{|c|}{$\begin{array}{cc}\text { 6-8090 } & \text { 6-8092.I } \\
\text { Davids Creek } & \text { Rast Nishnabotna River } \\
\text { near Hamlin } & \text { near Atlantic }\end{array}$} & \multicolumn{2}{|c|}{$\begin{array}{l}\text { 6-8095 } \\
\text { Tast Nishrsbotna River } \\
\text { at Red Oak. }\end{array}$} & \multicolumn{2}{|c|}{$\begin{array}{l}6.8100 \\
\text { Hishnabotna River } \\
\text { above Hamburg. }\end{array}$} & \multicolumn{2}{|c|}{$\begin{array}{l}\text { 6-8118.4 } \\
\text { Tarkio River } \\
\text { at Stanton }\end{array}$} \\
\hline & \multicolumn{2}{|c|}{$26.0 \mathrm{sq} \mathrm{mi}$} & \multicolumn{3}{|c|}{$432 \mathrm{sq} \mathrm{mi}$} & \multicolumn{2}{|c|}{$894 \mathrm{sq} \mathrm{mi}$} & \multicolumn{2}{|c|}{$2806 \mathrm{sq} \mathrm{ml}$} & \multicolumn{2}{|c|}{$49.3 \mathrm{sq} \mathrm{mi}$} \\
\hline & $h$ & B & & $A$ & $B$ & A & $B$ & $\grave{h}$ & $E$ & $\mathrm{~A}$ & $B$ \\
\hline $\begin{array}{l}0 \\
5 \\
10 \\
15 \\
20 \\
25 \\
30 \\
35 \\
40 \\
45 \\
50 \\
55 \\
60 \\
65 \\
70 \\
75 \\
80\end{array}$ & $\begin{array}{r}13.0 \\
10.0 \\
7.4 \\
5.4 \\
4.0 \\
2.9 \\
2.0 \\
1.4 \\
1.0 \\
.66 \\
.42 \\
.24\end{array}$ & $\begin{array}{r}13.0 \\
8.9 \\
5.9 \\
3.8 \\
2.4 \\
1.4 \\
.8 \\
.4 \\
.1\end{array}$ & & $\begin{array}{r}300 \\
230 \\
180 \\
142 \\
113 \\
91.0 \\
75.0 \\
62.0 \\
52.0 \\
44.5 \\
37.6 \\
32.4 \\
28.2\end{array}$ & $\begin{array}{r}300 \\
212 \\
153 \\
113 \\
83.0 \\
63.0 \\
48.5 \\
38.0 \\
30.0\end{array}$ & $\begin{array}{l}300 \\
2.57 \\
216 \\
130 \\
140 \\
121 \\
93.0 \\
79.0 \\
63.0 \\
50.0 \\
38.5 \\
29.5 \\
22.0\end{array}$ & $\begin{array}{l}300 \\
237 \\
186 \\
143 \\
108 \\
82.0 \\
60.5 \\
44.0 \\
31.0\end{array}$ & $\begin{array}{r}1000 \\
840 \\
710 \\
595 \\
500 \\
420 \\
358 \\
300 \\
255 \\
216 \\
184 \\
156 \\
133 \\
114 \\
97 . \\
84 . \\
72 .\end{array}$ & $\begin{array}{r}1000 \\
300 \\
640 \\
510 \\
410 \\
327 \\
260 \\
208 \\
166 \\
133 \\
106\end{array}$ & $\begin{array}{r}20.0 \\
13.4 \\
8.9 \\
5.8 \\
3.7 \\
2.3 \\
1.4 \\
. .83 \\
.48 \\
.27 \\
.15\end{array}$ & $\begin{array}{r}20 . \\
11 . \\
6 . \\
3 . \\
1 .\end{array}$ \\
\hline
\end{tabular}

* Use curve A when evapotranspiration losses are average or normal. Use curve $B$ during excessively hot, windy wedther. 
Table 1. Plotting data for base-flow recession curves -- continued

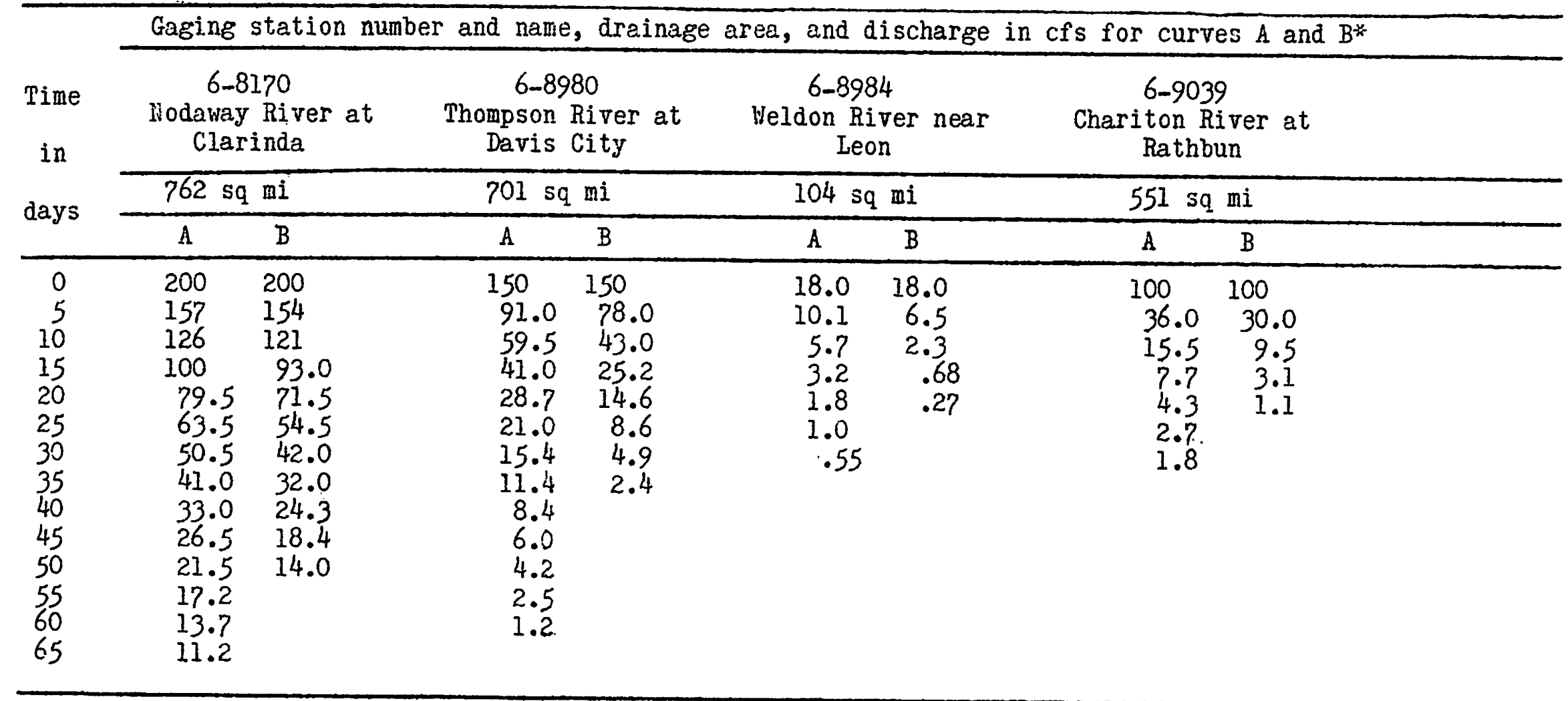

* Use curve A when evapotranspiration losses are average or normal. Use curve $B$ during excessively hot, windy weather. 